\title{
Kritische Infrastrukturen (KRITIS)
}

\section{KRITIS-Definition}

In einer modernen Gesellschaft ist die Nutzung diverser Informations- und Kommunikationsmittel nicht mehr wegzudenken. Das hat nicht zuletzt die derzeitige Pandemie gezeigt, in der in kürzester Zeit ganze Unternehmen und Behörden ins Homeoffice verlegt wurden. Doch ohne zum Beispiel dem notwendigen Strom, wären nicht nur der kommunikative Austausch, sondern zudem ganze Bereiche der industriellen Produktion lahmgelegt. Fehlt es zudem beispielsweise an einer Versorgung mit Trinkwasser, wäre ein Leben in den derzeitigen Formen kaum vorstellbar.

Unser tägliches Leben ist demnach vollständig durch den Einsatz moderne Technik und deren Funktionsfähigkeit geprägt. Um ordnungsgemäß zu funktionieren, ist wiederrum der Gebrauch bestimmter Basisdienste erforderlich. So können Kraftfahrzeuge und Heizanlagen ohne Treib- und Brennstoff nicht betrieben werden, Gebäude benötigen eine zuverlässige Energie- und Wasserversorgung und ohne ein Transportwesen kommen Waren und Dienstleistungen weder zur Fertigung noch zum Absatzmarkt. Diese für eine Gesellschaft bedeutsamen Basisdienste werden infolgedessen als Kritische Infrastrukturen (KRITIS) bezeichnet. Dies verdeutlich überdies die entsprechende Definition der Bundesregierung, wonach es sich bei Kritischen Infrastrukturen (KRITIS), um

„... Organisationen oder Einrichtungen mit wichtiger Bedeutung für das staatliche Gemeinwesen, bei deren Ausfall oder Beeinträchtigung nachhaltig wirkende Versorgungsengpässe, erhebliche Störungen der öffentlichen Sicherheit oder andere dramatische Folgen eintreten würden,"

handelt. ${ }^{1}$

\section{Einheitliche Sektoreneinteilung}

Bei Kritischen Infrastrukturen wird eine Aufteilung in Sektoren vorgenommen, auf die sich im Rahmen einer Bund-LänderArbeitsgruppe und einer Abstimmung auf Bundesebene verständigt wurde. ${ }^{2}$ Danach werden Organisationen und Einrichtungen aus den Bereichen Energieversorgung, Informationstechnik und

1 BMI, „Nationale Strategie zum Schutz Kritischer Infrastrukturen (KRITISStrategie)", 17. Juni 2009, S. 3 https://www.bmi.bund.de/SharedDocs/downloads/ $\mathrm{DE} /$ publikationen/themen/bevoelkerungsschutz/kritis.pdf?__blob=publicationFile\&v=3 (letzter Abruf 1.7.2021); dazu näher bei Mangels, DuD 2021, Heft 9, dieses Heft.

2 Siehe unter https://www.kritis.bund.de/SubSites/Kritis/DE/Einfuehrung/Sektoren/sektoren_node.html;jsessionid=0FAA9C866939920F72646647310D5997.2_ cid355 (letzter Abruf 26.7.2021)
Telekommunikation, Transport und Verkehr, Gesundheit, Wasser, Ernährung, Finanz- und Versicherungswesen, Staat und Verwaltung sowie Medien und Kultur zu den Kritischen Infrastrukturen gezählt, die aus verschiedenen Branchen bestehen. Im neuen IT-SiG 2.0 werden überdies zusätzlich die Sektoren Entsorgung und Unternehmen im besonderen öffentlichen Interesse (UNBÖFI) mit aufgenommen. ${ }^{3}$

Auf die jeweilige privatwirtschaftliche oder öffentlich-rechtliche Rechtsform kommt es in diesem Zusammenhang nicht an. Die Einteilung in Sektoren bildet vielmehr eine zwischen Bund und Ländern abgestimmte Grundlage für die Kooperation von Staat und Wirtschaft beim Schutz Kritischer Infrastrukturen. ${ }^{4}$

\section{Rechtliche Grundlagen}

Regelungen zur Kritischen Infrastruktur finden sich in unterschiedlichen Gesetzen bzw. Verordnungen. So richtet sich zum Beispiel die Definition Kritischer Infrastrukturen nach der BSIKritisverordnung (BSI-KritisV). ${ }^{5}$ Aufgrund der Einordnung als KRITIS-Unternehmen müssen Mindestanforderungen an die ITSicherheit erfüllt und IT-Sicherheitsvorfälle an das Bundesamt für Sicherheit in der Informationstechnik (BSI) gemeldet werden. ${ }^{6}$ Bei der Festlegung geeigneter technischer und organisatorischer Maßnahmen ist dabei der „Stand der Technik“ zu beachten (vgl. IT-SiG). ${ }^{7}$ In Kürze wird das IT-SiG 2.0 erwartet, welches zusammen mit der BSI-KritisV 2.0 die KRITIS-Regulierung voraussichtlich erweitert, sodass nach derzeitigem Diskussionstand mehr Pflichten für die Betreiber und ein Anstieg der Befugnisse für den Staat zu erwarten sind. ${ }^{8}$

3 Ausführlich bei Woitke, DuD 2021, Heft 9, dieses Heft.

$4 \mathrm{Vgl}$. insgesamt unter https://www.kritis.bund.de/SubSites/Kritis/DE/Einfuehrung/Sektoren/sektoren_node.html;ssessionid=0FAA9C866939920F72646647310D 5997.2_cid355 (letzter Abruf 31.7.2021).

5 BMJV, „Verordnung zur Bestimmung Kritischer Infrastrukturen nach dem BSI-Gesetz (BSI-Kritisverordnung - BSI-KritisV)", 22. April 2016, https://www.gesetze-im-internet.de/bsi-kritisv/BJNR095800016.html (letzter Abruf 26.7.2021).

6 Zur Notwendigkeit diese zu prüfen, vgl. bei Stradomsky, DuD 2021, Heft 9, in diesem Heft.

7 Vgl. BSI, „Rechtsgrundlagen: NIS-Richtlinie, IT-Sicherheitsgesetz, BSI-Gesetz, BSI-Kritisverordnung", 2021, https://www.bsi.bund.de/DE/Themen/KRITISund-regulierte-Unternehmen/Kritische-Infrastrukturen/Allgemeine-Infos-zuKRITIS/Rechtsgrundlagen/rechtsgrundlagen_node.html; OpenKRITIS, „KRITIS-Gesetzgebung", 2021, https://www.openkritis.de/it-sicherheitsgesetz/ gesetzgebung-kritis-bsig.html (letzter Abruf 26.7.2021); auführlich dazu bei Woitke, DuD 2021, Heft 9, dieses Heft; die Sektoren Medien und Kultur sowie Staat und Verwaltung werden nicht durch das IT-Sicherheitsgesetz (IT-SiG) reguliert.

8 Vgl. Mangels, DuD 2021, Heft 9, dieses Heft; Kipker/Scholz, DuD 2021, S. 40. 